\title{
Putting microfluidics in other people's hands
}

\author{
In microfluidics, sharing is hard. But practitioners are exploring new ways to share designs, devices and \\ experience.
}

It's designed, built, validated: it's time for your lab to celebrate the birth of a microfabricated device that can be used for bioanalysis or manipulation experiments and needs only microliters, or even picoliters, of reagents. The after-party for this new microfluidic device is about sharing. Full-steam ahead with experiments, a manuscript including the device design, a device-building protocol as a document, a web page, a video or all of these.

You daydream as the milk swirls into your coffee. Upon publication of your paper, many colleagues in your subfield reach out, eager to try your device. The sugar crystals pinging the sides of the coffee cup sound like clinking coins after your imagined phone call with a venture capital firm about your device's commercial potential. Then the postdoc comes over to show you something, the daydream evaporates, and a real day begins. Building a reproducible microfluidic device can be a pipe dream. We asked some practitioners how to improve some of the routes for microfluidic sharing. For some it's building ways to share designs and construction experiences, for others it's taking the leap to commercialization,

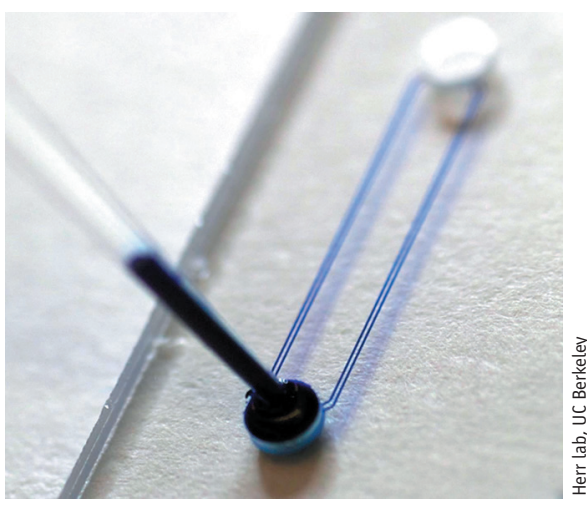

A glass microfluidic device for separating and probing protein isoforms. Reproduced with permission from ref. 7.

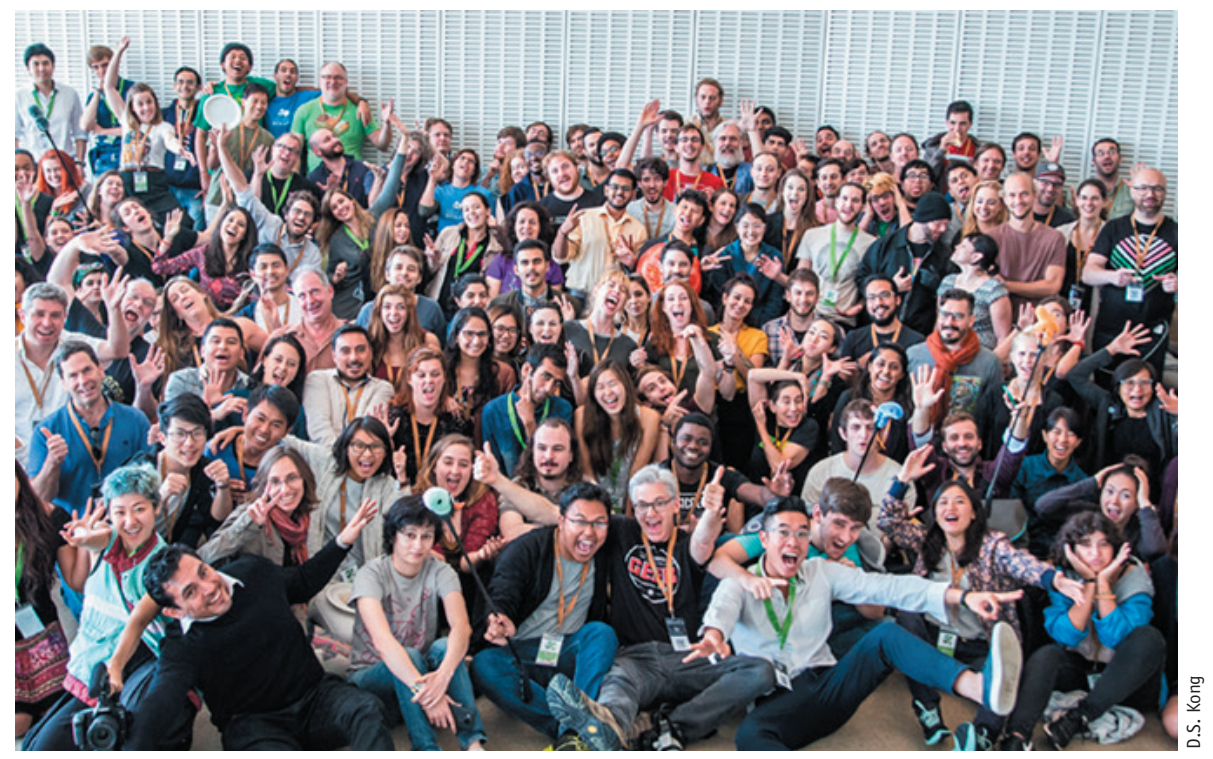

Sharing culture in microfluidics will help the field. Bio-hackers of many stripes got together at the Global Community Bio Summit.

and others hunt for ways to share a 'mother wafer'.

\section{A sharing culture}

To obtain training directly from device and tool developers, people with some experience in microfluidics can take 'Sunday' workshops at microfluidics conferences such as microTAS, says Amy Herr, bioengineer and microfluidic device developer at the University of Berkeley. There are general workshops, such as the upcoming 'How to Make It and Use It' at the University of Kansas. There are summer schools. At Cold Spring Harbor Laboratory, she co-teaches the summer course Single Cell Analysis, where scientists from around the world learn to fabricate devices, complete assays and analyze data. The course helps users and shows device and assay developers how life scientists interpret a protocol or react to a tool, and it gives them ideas about future devices.
Through community sites such as Metafluidics.org, users can now share the designs of their microfluidic devices and device parts ${ }^{1,2}$. Scientists at MIT Lincoln Laboratory launched the portal in the spring of 2017. The founder group is led by bioengineer David Kong, who has left Lincoln Lab to run Metafluidics.org as part of his Community Biotechnology Initiative at the MIT Media Lab. Regular videoconferences draw participants from the now 1,000 members of the Metafluidics global community.

This resource is part of the emerging, larger do-it-yourself culture in science, says Peter Carr, a Lincoln Lab synthetic biologist, bioengineer and Metafluidics co-founder. He and Kong worked together on microfluidic gene synthesis projects in Joe Jacobson's group at the Media Lab. They like community biolabs and makerspaces where scientists and citizen scientists tinker, learn and gain access to prototyping tools. For example, in the 1,000 labs in nearly 80 countries 


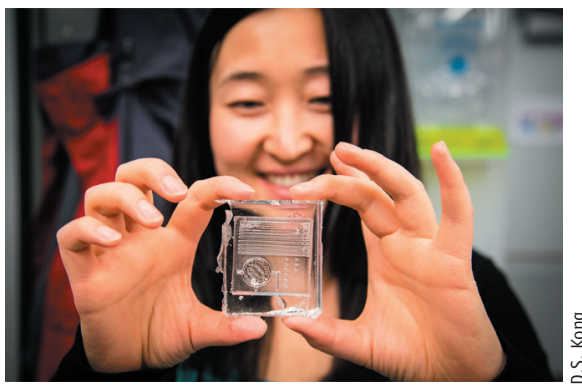

MIT Media Lab graduate student Lining Yao with a device she has made.

in the Fab Lab Network, people can make microfluidic devices at low cost.

Microfluidics has originated in labs where production and use can involve an "artistry component," says Carr. A device might work only after the 'special touch' from a few people or even one person in the lab, and 'on-the-fly' adjusting is common. The DIY movement can help the field grow and share and he hopes to help infuse it with established engineering principles.

\section{Commercial sharing}

UC Berkeley's Herr has spun two companies out of her lab, Zephyrus Biosciences and Correlia Biosystems, which is one way of getting microfluidic systems into the hands of people needing these tools, she says. The companies are also a potential career path for her lab's alumni. UC Berkeley helps startups with, for example, mentorship or advice on technology transfer, and she is glad to be immersed in the San Francisco Bay area's startup-friendly culture. Many of these resources can benefit scientists elsewhere, she says.

Biologist Jonathan Thon feels ready to commercialize his microfluidic approach. During his postdoctoral fellowship at Brigham and Women's Hospital and Harvard Medical School, Thon studied how specialized cells in the bone marrow called megakaryocytes produce plateletslong a focus of his mentor Joseph Italiano. To address the shortage of blood donors the researchers now want to make human platelets on a large scale, in microfluidic bioreactors from megakaryocytes derived from induced pluripotent stem cells. The microfluidics - a proprietary design and build-imitate the shearing forces of the bone marrow environment.

The researchers spun out a company, Platelet BioGenesis, which set up shop in an incubator of the University of Massachusetts, Boston, then moved to one in Cambridge run by the venture firm Nest.Bio. Last year, after raising \$10 million in venture capital financing, Thon decided to become the company's full-time CEO and chief scientific officer. Internal validation of the platform is completed and the company is now exploring scale-up, which includes producing many devices and other steps, all done with an eye to regulatory review.

The decision to commercialize one's microfluidic platform and live a startup life involves much uncertainty, says Thon. It takes a deep feeling that a problem matters and that you see a path to a viable solution. Most importantly, the process takes drive. Focusing too much on the risk "can very quickly lead to crushing despair," he says. "It's best to not go down that path." The process is uncontrollable in many ways. "What you can control is a clear vision for what is needed and the drive to see it through, whatever shape that takes," he says. With a working microfluidic device, a scientist will want to remember that motivation and the purpose the device serves. For him, the microfluidic device connects research and development with platelet manufacture. When setting out, he says, one has to plot out the required infrastructure, the gaps that need to be bridged, and it's important to situate oneself in the venture. "The exercise should absolutely scare you," says Thon.

Were Thon a microfluidic device developer trying to find users, a few things that would help, he says, are on-site foundries at universities, standard and transparent material transfer agreements, stable manufacturing and quality control metrics that enable continuity of use. Also helpful: a way to collect metrics of interest, to determine when applying a device needs further internal development or when it's time for external input.

MicroLIQUID, a Spanish company near San Sebastian in Basque Country, was spun

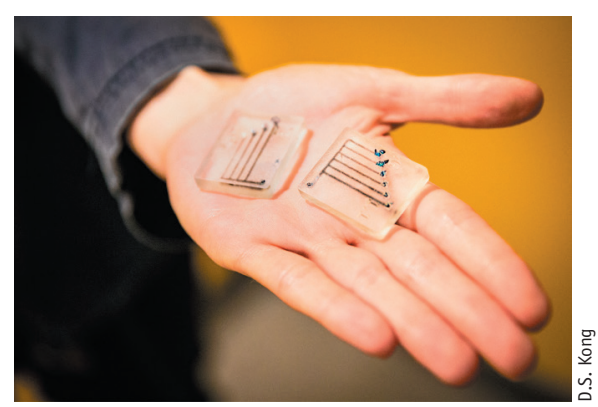

With Metafluidics.org and other initiatives, the initiators hope to turn some artisanal aspects of microfluidics into more reproducible engineering.

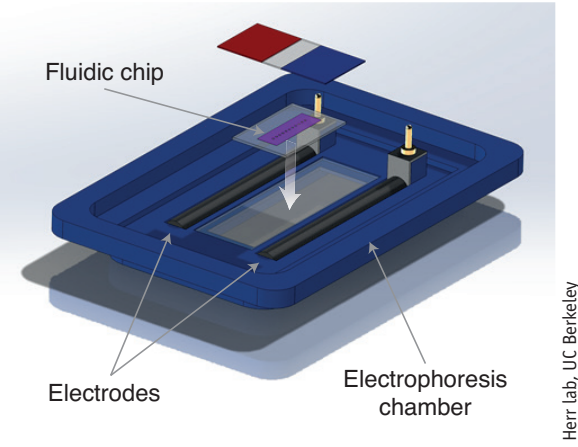

In a fluidic chip the size of a microscope slide, cells are lysed and electrophoresis is run on proteins from the nucleus and the 14 picoliters of cytoplasm $^{8}$.

out of technology incubator IKERLAN in 2008, says Francisco Blanco, a bioengineer and company co-founder who leads the company's business development and innovation. IKERLAN is part of a larger Basque government effort to support technology, including microsystems. MicroLIQUID's first customers were mainly academics requesting microfluidic chip development. Today, around $15 \%$ of customers are academics, and the rest are pharmaceutical and biotech companies, of which around $55 \%$ are in Europe and around $40 \%$ are in the US. Blanco likes working with academics because of the way it drives his company toward next-generation devices. In his view, such collaborations need to be done without obsessing over next quarter's financial results.

In 2013, the company switched from making stand-alone chips to integrating microfluidic chips into instruments, says Blanco. "It was and is a challenge," he says, but it reflects customer needs. It plays out well because it's hard to launch a microfluidic device without knowing the automated instrumentation it will be associated with. That can help to explain why plenty of microfluidic chips do not make it to market even after many years in development.

Herr is supportive of startup ambitions in her lab. As a background project, the team found that over 300 companies use microfluidics in their products. But her focus on bioanalytical methods including microfluidics is ensuring that her trainees get rigorous academic experience where they learn, grow and contribute. She is uncomfortable with encouraging students to work on a startup before the end of their training is in sight. "Others disagree on this, but my first goal is their training," she says. Next is to 
help them launch a successful, productive career.

In the context of commercialization, Herr often hears scientists say they want to do something "besides just publishing papers." Such comments "belittle the important role of 'just publishing,' " she says. Publishing via peer review is creating and sharing knowledge, which is "essential for humankind" and leads toward future understanding of many questions. The two avenues are not mutually exclusive but she 'jumps in' when a researcher belittles the notion of "just" publishing papers. "We need both," she says.

\section{Academic sharing}

University of Groningen researcher Matthias Heinemann faces a nail-biter situation. $\mathrm{He}$ has only one silicon wafer with which to make microfluidic devices. It's nine years old, which, in wafer years, is a geological time frame. With age, it has become slightly damaged, so it's only half a wafer that five people in his lab share. They rely on it for making devices for their daily experiments. It's the only one that leads, via soft lithography, to well-working microfluidic devices. "My nightmare is that if this would now break, then half of my lab gets stuck," he says. He would like to share copies of the wafer with external scientists. But he doesn't have those.

The device works for the Heinemann lab's single-cell experiments about yeast metabolism, to learn, for example, about factors influencing life $\operatorname{span}^{3,4}$. Some labs, using different microfluidic devices, have found that caloric restriction extends yeast life span. "When we tried this in our microfluidic device, we couldn't see this," he says. Plenty of reasons, including different protocols, dissimilar device designs and builds, likely con-

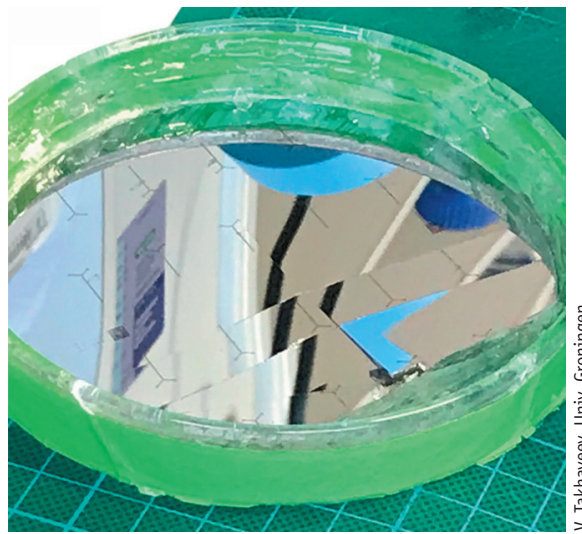

It's nine years old, a bit broken, but this wafer makes microfluidic devices that work, says Matthias Heinemann.

\section{BOX 1 SOFT LITHOGRAPHY AT WORK}

A microfluidic device is built from a silicon wafer with a 'master mold' of the desired device design.

- Device design features are embossed onto the wafer surface using a photomask and a light-sensitive polymer.

- A spoonful of another polymer is added, and the wafer is spin-coated.

- Heat cures the polymer to the mold's microscale features.

- The now rubbery polymer can be peeled off the wafer.

- Other layers can be added and connections between layers, such as valves, are punched in.

- The microfluidic device is annealed to a glass slide.

Source: A. Herr, UC Berkeley

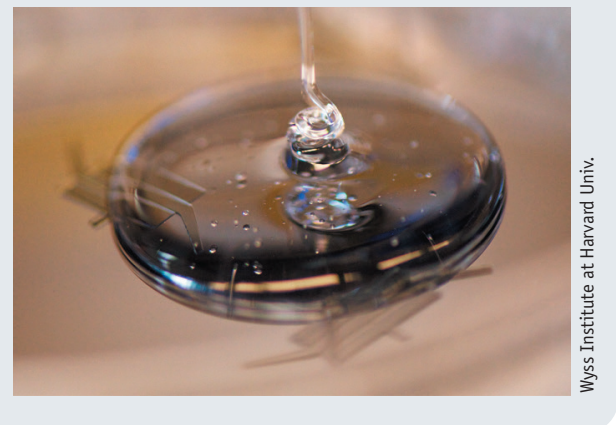

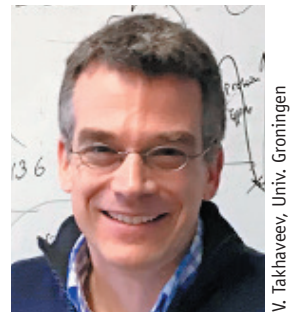

Matthias Heinemann hopes for a "universal supplier" of validated microfluidic devices.

bricates microfluidics full time at the ETH's microscopy core facility, has tried to make another such wafer, and failed. Heinemann collaborated with MicroLIQUID but the devices the company made didn't work as well as ones made from the original wafer. Blanco acknowledges this and says that in the company's early years, the team was setting up internal standards and the company is still learning. $\mathrm{He}$ and his team work through failures, sometimes investing in new equipment to be ready for the next wave of microfluidic devices.

Perhaps the first wafer was a lucky fluke, says Heinemann. He uses it and worries. Even with simple designs, if one tiny pillar in the microfluidic device is a micron too low, too many cells get trapped and experiments can stall. Even simple microfluidics design can be hard to replicate. "If you compare it with Steve Quake's designs, it's not even kindergarten design," he says, referring to the microfluidics pioneer Stanford University researcher Stephen Quake.
A "magic mold" is not uncommon, says Carr. "That story does not surprise me at all." Sources of microfluidic device variability include batch-to-batch variability with the elastomer polydimethylsiloxane (PDMS), the length of time polymer and initiator are mixed, and uneven temperatures when the elastomer is cured onto the wafer (see Box 1, "Soft lithography at work").

As Blanco explains, his company spent a year and a half prototyping microfluidic designs for the lab of University of Zaragoza researcher Rosa Monge, who uses microfluidics to model processes in glioblastoma. "Reproducibility was the main challenge," he says. "Their patience was very important." Monge has founded a company, BEOnChip, and is collaborating with MicroLIQUID to scale up manufacture of the devices and broaden the cancer types they can be used for.

For Heinemann, no wafer is as good as the 'mother wafer'. The lab continues work with its half a wafer, enabled by devices made of PDMS, which is transparent and thus microscopy-ready ${ }^{5}$. But PDMS has its challenges.

\section{Material matters}

PDMS can interact with small molecules and wreak havoc in basic research projects. A few years ago, as part of his work on metabolism, Heinemann and his team did experiments in his device using smallmolecule inhibitors, but saw no inhibitory effect. The inhibitor was getting stuck to the PDMS. "It never reached the cells," he says. Microfluidics certainly enables many types of experiments but in some cases, "you need to think about different materials." 
In the case of Platelet BioGenesis, says Thon, the reactor must support sufficient numbers of megakaryoctyes for preclinical work to begin. One megakaryocyte can deliver around 100 platelets. He and his team are assessing platelet quality and safety in vitro and in vivo. He confirms that PDMS can leach substances that can be toxic to cells and can absorb small molecules, hindering drug studies. There are alternate materials, he says. For platelet bioreactors the firm might, for example, consider polycarbonate, polyolefin or graphene oxide.

One option, says Thon, is for a researcher to first build a PDMS-based device and switch material in scale-up. He or she might have device design expertise and knowledge of small-scale or small-batch PDMS manufacture and biomaterials. But industrial scaleup of cell-culture manufacturing, translation of processes for regulatory approval, largescale manufacturing and quality assessment are not typically in a researcher's wheelhouse, he says. That's when it's a good idea to reach out to consultants; more advisors will emerge as the field grows. "Most often you'll need to solicit advice from a number of different people, each with their own piece of the puzzle, to put the full picture together." Design for manufacturability is 'productization', says Herr, whose lab does not use much PDMS. But in early-stage research, she and her team do not focus on manufacturing issues. They revisit materials questions later in development.

\section{Sharing virtues}

Once a device has been built and tested, a lab will want to share the design with others. The best way to share, says Herr, is to provide detailed and complete information in peer-reviewed publications. When it makes sense, labs might prepare a paper devoted to the protocol. Her microfluidic devices, which include an approach for single-cell western blotting ${ }^{6}$, are made in her lab and the UC Berkeley nanofabrication facility.

Metafluidics.org, the platform devoted to sharing microfluidic design, is a "great idea," she says. She includes design materials such as those on the site in supplementary materials to papers. MicroLIQUID's Blanco likes the site, too, and says he is personally passionate about such initiatives. The company's thousands of designs cannot be shared because of customer intellectual property provisions. But his company is exploring how it can take part in microfluidic device design sharing to

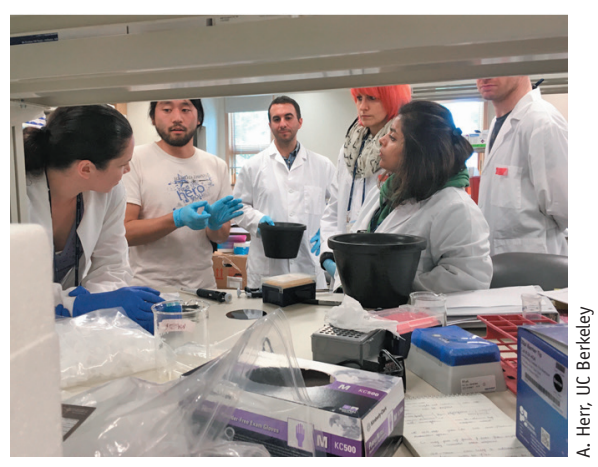

is to cultivate an engaged global community of developers and users to lower the barriers of access to fluidics," says Kong.

The community-organizing exercise includes academic communities as well as participants from the global maker and biomaker movements; Metafluidics has academic as well as non-expert users. "This for me is particularly exciting, seeing novices get into the field," says Kong. He believes in the power of this diversity to drive innovation. By his estimation, in the microfluidics field,

In summer courses such as this one at Cold Spring Harbor Laboratory, students learn to fabricate microfluidic devices and complete assays.

help "push this amazing field to more and more solutions for our society."

Design file-sharing is sometimes not enough, says Herr, which is, for example, where the Journal of Visual Experiments can be helpful. Kong hopes that journals will offer more rigorous documentation on how to produce a fluidic system from a research paper. That, he says, "would be a significant improvement over the status quo," given that all too often critical details such as design files are absent.

Ideally, says Kong, sharing design files will become a more standardized practice, as would more standardized documentation, including how-to videos and tutorials. "It would be wonderful for editors of journals to establish a culture of sharing and using infrastructure like Metafluidics," he says. As Carr points out, Lincoln Lab mainly does research for the US Defense Department, but he is happy the lab gave Metafluidics its startup funding and support to help enable labs to reproduce the work of others.

As Kong explains, in addition to global video conferences, he wants to grow the community and excitement about opensource microfluidics. Metafluidics.org is the hardware portal for the Living Computing Project, funded by the National Science Foundation, which is about 'programing biology': bringing to bio-materials and therapeutics development, for example, the broad approach, specification and design advances that have taken place in computing with people of many skill levels building tools and apps. This year he has a collaboration with the Global Community Bio Summit and the Gathering of Open Source Hardware in the works. "While Metafluidics itself is a useful piece of infrastructure for sharing device designs, its ultimate purpose only a tiny fraction of published microfluidic devices end up being used by others. That's why Metafluidics aims to support device reproduction, and he is glad to see some community members reproducing designs they find on the site.

Heinemann, who shares his designs in papers and via e-mail, likes the idea of Metafluidics. He also hopes for a "universal supplier" of validated microfluidic devices. "Having a fabrication facility coupled with Metafluidics would be a wonderful advance," says Kong. In the Metafluidics community there are 'microfluidic nerds' invested in the nuances of device design and remixing, while others are users who just want the device and don't want to have to manufacture it themselves.

The reproducibility issue Heinemann describes "is a big one for the field," says Kong. "There are real microfluidic artisans out there, and even they may have difficulty reproducing the 'workhorse wafer' that makes the devices just right." He and his team are setting up a forum for Metafluidics, where the community can interact and share. The idea, he says, is to help convert some of the artisanal aspects of fluidics into more reproducible engineering practice.

Vivien Marx is technology editor for Nature Methods (v.marx@us.nature.com)

1. Walsh, D.I. 3rd, Kong, D.S., Murthy, S.K. \& Carr, P.A. Trends Biotechnol. 35, 383-392 (2017).

2. Kong, D.S. et al. Nat. Biotechnol. 35, 523-529 (2017).

3. Papagiannakis, A., Niebel, B., Wit, E.C. \& Heinemann, M. Mol. Cell. 65, 285-295 (2017).

4. Huberts, D.H. et al. Proc. Natl. Acad. Sci. USA 111, 11727-11731 (2014).

5. McDonald, J.C. et al. Electrophoresis 21, 27-40 (2000).

6. Hughes, A.J. et al. Nat. Methods 11, 749-755 (2014).

7. Hughes, A.J., Lin, R.K., Peehl, D.M. \& Herr, A.E. Proc. Natl. Acad. Sci. USA 109, 5972 5977 (2012).

8. Yamauchi, K.A. \& Herr, A.E. Microsyst. Nanoeng. 3, 16079 (2017). 